\title{
Optimal field-free molecular orientation with nonresonant two-color adiabatic-turn-on and sudden-turn-off laser pulses
}

\author{
Wang, Shuo; Henriksen, Niels Engholm
}

Published in:

Physical Review A

Link to article, DOI:

10.1103/PhysRevA.102.063120

Publication date:

2020

Document Version

Publisher's PDF, also known as Version of record

Link back to DTU Orbit

Citation (APA):

Wang, S., \& Henriksen, N. E. (2020). Optimal field-free molecular orientation with nonresonant two-color adiabatic-turn-on and sudden-turn-off laser pulses. Physical Review A, 102(6), [063120].

https://doi.org/10.1103/PhysRevA.102.063120

\section{General rights}

Copyright and moral rights for the publications made accessible in the public portal are retained by the authors and/or other copyright owners and it is a condition of accessing publications that users recognise and abide by the legal requirements associated with these rights.

- Users may download and print one copy of any publication from the public portal for the purpose of private study or research.

- You may not further distribute the material or use it for any profit-making activity or commercial gain

- You may freely distribute the URL identifying the publication in the public portal

If you believe that this document breaches copyright please contact us providing details, and we will remove access to the work immediately and investigate your claim. 


\title{
Optimal field-free molecular orientation with nonresonant two-color adiabatic-turn-on and sudden-turn-off laser pulses
}

\author{
Shuo Wang $\oplus^{1}$ and Niels E. Henriksen $\oplus^{2, *}$ \\ ${ }^{1}$ School of Physics, Dalian University of Technology, Dalian 116024, People's Republic of China \\ ${ }^{2}$ Department of Chemistry, Technical University of Denmark, Building 206, DK-2800 Kongens Lyngby, Denmark
}

(Received 30 October 2020; accepted 10 December 2020; published 28 December 2020)

\begin{abstract}
Field-free molecular orientation, achieved by using two-color slow turn-on and rapid turn-off laser pulses, is theoretically investigated for linear molecules. We derive analytical results for the maximum degree of field-free orientation for a given number of populated rotational states. We show that numerical results with adiabatic-turnon and sudden-turn-off laser pulses attain the optimal orientation. Based on the adiabatic theorem, the effects of laser and molecular parameters on both the adiabatic process and the degree of molecular orientation are analyzed. The adiabaticity is well reflected through the temporal evolution of the phase difference between two adjacent rotational states.
\end{abstract}

DOI: 10.1103/PhysRevA.102.063120

\section{INTRODUCTION}

The technology of controlling molecular alignment and orientation, based on its highly anisotropic characteristic, has been used in many fields. Ionization probabilities, which are important for understanding strong-field molecular physics, can be enhanced by aligning molecules [1]. Spatially aligned and oriented molecules are very important for investigating in detail the stereodynamics of molecules [2,3]. Enhanced high-order harmonic generation widely used in molecular imaging [4], nuclear dynamics retrieving [5], and attosecond pulse production [6], was observed experimentally in aligned molecular systems [7].

Many studies have been carried out theoretically and experimentally to achieve a high degree of molecular alignment or orientation [8]. All methods can be roughly divided into two strategies with adiabatic or nonadiabatic laser-molecule interactions. Molecular alignment is obtained mainly by the interaction of laser fields and molecular polarizability. Adiabatic alignment was proposed and observed after the slow turn-on of an intense laser field [9-11]. At the same time several nonadiabatic methods with sudden pulse interaction were introduced which are capable of producing field-free alignment [12-14]. The degree of alignment depends on the population as well as the phases of even rotational states. Almost the same even rotational state phases can be obtained after an adiabatic process, while a broad rotational state distribution can be obtained after a sudden pulse but phases are very different [15].

In order to obtain molecular orientation, there are higher requirements for the laser pulse to make sure that odd rotational states also are populated. A nonresonant two-color femtosecond laser was used to interact with the molecular hyperpolarizability in order to break the parity of the rota-

\footnotetext{
*neh@kemi.dtu.dk
}

tional wave function [16,17]. Alternatively, a THz pulse was used to generate molecular orientation by permanent dipole interaction $[18,19]$. Considering the limit of THz pulse intensity, highly excited rotational states are not easily populated. Subsequently, some combination schemes were proposed to get a high degree of molecular orientation [20-23]. As in the case of alignment, it is difficult to keep the phase difference of adjacent rotational states identical for the above mentioned nonadiabatic orientation process which limits acquirement of a higher degree of orientation [24].

One method significantly enhanced the degree of orientation by combining a nonresonant laser with sufficiently long duration and a weak electrostatic field [25,26]. Compared to the molecular alignment, adiabatic orientation is more difficult to fulfill in this mixed-field method. The dependence on intensity and duration of laser pulses and the strength of the electrostatic field which influence the adiabatic process has been discussed in detail [27]. Meanwhile, experiments based on the mixed-field have been conducted [28-30].

Considering the advantages of both adiabatic and nonadiabatic methods, a slow turn-on and sudden-turn-off truncated laser pulse was proposed to significantly increase the degree of alignment [31,32]. Likewise, an approach to achieve field-free molecular orientation was demonstrated theoretically and experimentally with a two-color slow turn-on and rapid turn-off laser [33-36]. The general consensus on achieving adiabatic conditions is that the rise time of the laser pulse should be much longer than the rotational period of the molecules. Intensities and delay of two-color truncated pulses have been optimized to achieve an adiabatic rotational wave packet evolution [37].

In addition to the laser parameters, the molecular parameters, such as rotational constant, polarizability and hyperpolarizability, influences the realization of adiabatic processes and the degree of molecular orientation. All the parameters need to be taken into account to determine the optimal laser parameters for a specific molecule or the optimal 
molecules for a laser pulse with a specific energy. In this paper, two-color truncated pulses are used to control the field-free orientation of linear molecules. How the parameters of laser pulses and molecules affects the adiabatic process is discussed based on the adiabatic theorem. The temporal evolution of the two parts in the adiabatic theorem, the rate of change of the Hamiltonian and the instantaneous energy difference, are shown separately. It gives an intuitive picture of the dynamics. Further, a two-state model is used to explain the results.

\section{THEORY}

\section{A. Model and basic equations}

We consider a molecule described as a linear rigid-rotor subject to two-color linearly polarized nonresonant laser pulses with slow turn-on and rapid turn-off. The polarization axis of the pulse is defined as the laboratory-fixed $Z$ axis. The form of the laser pulse is expressed as

$$
E(t)=\varepsilon(t)[\cos (\omega t)+\cos (2 \omega t)]
$$

with

$$
\varepsilon(t)=E_{0} \exp \left(-\frac{t^{2}}{2 \sigma^{2}}\right)\left\{\begin{array}{l}
\sigma=\sigma_{R}(t \leqslant 0) \\
\sigma=\sigma_{F}(t>0)
\end{array},\right.
$$

where $\omega$ is the fundamental frequency of the laser pulse, $\varepsilon(t)$ is a Gaussian shape envelope of $E(t), E_{0}$ is the peak amplitude of the laser, $\sigma$ is related to the FWHM (FWHM $=2 \sigma \sqrt{2 \ln 2}$ ), $\sigma_{R}$ and $\sigma_{F}$ determines the rise and fall time, respectively.

Assuming that the frequency of the laser is much larger than the rotational frequency of the molecule, after applying the rotating wave approximation, the Hamiltonian of a linear molecule irradiated by a two-color laser pulses is given by [8]

$$
\begin{aligned}
\hat{H}(t)= & B \hat{J}^{2}-\frac{\varepsilon^{2}(t)}{2} \Delta \alpha \cos ^{2} \theta \\
& -\frac{\varepsilon^{3}(t)}{8}\left[\left(\beta_{\|}-3 \beta_{\perp}\right) \cos ^{3} \theta+3 \beta_{\perp} \cos \theta\right]
\end{aligned}
$$

with $B$ the rotational constant of the molecule, $\hat{J}^{2}$ the squared angular momentum operator, $\theta$ the angle between the laser polarization and the molecular axis, $\Delta \alpha$ the anisotropic polarizability, and $\beta_{\|}$and $\beta_{\perp}$, the hyperpolarizability components parallel and perpendicular to the molecular axis, respectively. It can be seen from Eq. (3), that the polarizability term forms a symmetric double-well potential in $\theta$ space between $-\pi / 2$ to $3 \pi / 2$ and the hyperpolarizability term leads to an asymmetry of the double-well potential.

We start by formulating the dynamics in terms of the density matrix method but will eventually use the Schrödinger picture since we only consider pure quantum states. The density operator can be expanded in eigenstates of a rigid rotor in a field-free space as

$$
\hat{\rho}(t)=\sum_{J, M, J^{\prime}, M^{\prime}} \rho_{J, M, J^{\prime}, M^{\prime}}(t)|J, M\rangle\left\langle J^{\prime}, M^{\prime}\right|
$$

The time evolution of the density operator is determined by the Liouville equation. A fourth-order Runge-Kutta method is used to solve the differential equations [38]. The degree of molecular orientation is given by

$$
\begin{aligned}
\langle\cos \theta(t)\rangle= & \operatorname{Tr}\{\cos \theta \hat{\rho}(t)\} \\
= & \sum_{J^{\prime \prime}, M^{\prime \prime}}\left\langle J^{\prime \prime}, M^{\prime \prime}|\cos \theta \hat{\rho}(t)| J^{\prime \prime}, M^{\prime \prime}\right\rangle \\
= & \sum_{J, M}\left(\rho_{J+1, M, J, M}(t)+\rho_{J+1, M, J, M}^{*}(t)\right) \\
& \times\langle J+1, M|\cos \theta| J, M\rangle .
\end{aligned}
$$

The phase difference between two adjacent rotational states $\phi_{J+1, J}(t)$ can be calculated based on the real and imaginary part of $\rho_{J+1, M, J, M}(t)$.

In this paper, we mainly investigate the dynamics based on the adiabatic theorem [39]. If the condition

$$
\left|\hbar\left\langle m\left|\frac{\partial \hat{H}}{\partial t}\right| n\right\rangle /\left(E_{n}-E_{m}\right)^{2}\right| \ll 1
$$

is satisfied, the dynamics is adiabatic. $|m\rangle$ and $|n\rangle$ are the instantaneous eigenstates of $\hat{H}(t) . E_{m}$ and $E_{n}$ are the corresponding instantaneous eigenvalues. The instantaneous eigenvalues and eigenstates in the $|J, M\rangle$ representation at a certain time can be calculated based on the matrix of $\hat{H}(t)$ in the $|J, M\rangle$ representation. The rate of change of the Hamiltonian is given by

$$
\begin{aligned}
\frac{\partial \hat{H}}{\partial t}= & \Delta \alpha \cos ^{2} \theta \varepsilon^{2}(t) \frac{t}{\sigma^{2}} \\
& +\frac{3}{8}\left[\left(\beta_{\|}-3 \beta_{\perp}\right) \cos ^{3} \theta+3 \beta_{\perp} \cos \theta\right] \varepsilon^{3}(t) \frac{t}{\sigma^{2}} .
\end{aligned}
$$

Then we can evaluate if the process is adiabatic by comparing $|\langle m|\partial \hat{H} / \partial t| n\rangle|$ and $\left|\left(E_{n}-E_{m}\right)^{2} / \hbar\right|$, the former represents the coupling matrix element of $\partial \hat{H} / \partial t$ and the latter represents the corresponding instantaneous energy difference. Both dimensions are energy/time.

The instantaneous eigenstates can also be converted from the $|J, M\rangle$ representation to a representation in terms of spherical harmonics by

$$
\begin{aligned}
\langle\theta, \phi \mid n(t)\rangle & =\sum_{J, M} C_{J, M}^{(n)}(t)\langle\theta, \phi \mid J, M\rangle \\
& =\sum_{J, M} C_{J, M}^{(n)}(t) Y_{J}^{M}(\theta, \phi) \quad(t<0)
\end{aligned}
$$

with $C_{J, M}^{(n)}$ being the complex coefficient of the $|J, M\rangle$ state of the $n$th instantaneous eigenstate, which gives us a more intuitive picture of molecular orientation.

\section{B. Estimation of the maximum degree of orientation}

After the laser pulse, molecules return to free-rotor states, then we have

$$
\begin{aligned}
\langle\cos \theta(t)\rangle= & 2 \sum_{J=0}^{J_{\max -1}} \sum_{M=-J}^{J}\left|C_{J+1, M} C_{J, M}\right| \\
& \times\langle J+1, M|\cos \theta| J, M\rangle \\
& \times \cos \left(\frac{\Delta E_{J+1, J}}{\hbar} t-\varphi_{J+1, J}\right),
\end{aligned}
$$


where $J_{\max }$ is the highest rotational state in the sum, $C_{J, M}$ is the complex coefficient of the rotational state, $\Delta E_{J+1, J}$ and $\varphi_{J+1, J}$ are the energy difference and phase difference between two adjacent rotational states after the decay of the pulse. We observe that for a given population of the rotational states, the highest degree of orientation is obtained when $\varphi_{J+1, J}$ is constant, i.e., independent of $J$.

Based on the method of Lagrange multipliers, the maximum degree of orientation can be estimated for different $J_{\max }$ (for related previous work, see Ref. [40]). The matrix elements in Eq. (9) $\langle J+1, M|\cos \theta| J, M\rangle=(J+1) /$ $\sqrt{(2 J+3)(2 J+1)}$ (for $M=0)$ are denoted by $M_{J+1, J}$, then the Lagrange function can be written as

$$
\mathcal{L}\left(\left|C_{0}\right|,\left|C_{1}\right|, \ldots,\left|C_{J_{\max }}\right|, \lambda\right)=f-\lambda g
$$

where

$$
\begin{aligned}
& f=2 \sum_{J=0}^{J_{\max }-1}\left|C_{J+1} C_{J}\right| M_{J+1, J}, \\
& g=\sum_{J=0}^{J_{\max }}\left|C_{J}\right|^{2}-1=0 .
\end{aligned}
$$

$f$ is the degree of orientation at revival times when the phase difference $\varphi_{J+1, J}$ is constant, $\lambda$ is the Lagrange multiplier, and $g$ is the constraint. To find the extrema of $f$ subject to $g$, we need $\nabla \mathcal{L}=0$, then we have

$$
\begin{array}{r}
M_{1,0}\left|C_{1}\right|-\lambda\left|C_{0}\right|=0, \\
M_{1,0}\left|C_{0}\right|+M_{2,1}\left|C_{2}\right|-\lambda\left|C_{1}\right|=0, \\
M_{2,1}\left|C_{1}\right|+M_{3,2}\left|C_{3}\right|-\lambda\left|C_{2}\right|=0, \\
\cdots \\
M_{J_{\max }, J_{\max }-1}\left|C_{J_{\max }-1}\right|-\lambda\left|C_{J_{\max }}\right|=0 .
\end{array}
$$

Nontrivial solutions are obtained when the determinant of the coefficients is equal to zero, which determines $\lambda$. By multiplying each equation in Eq. (12) by the corresponding coefficient $\left|C_{J}\right|$ of $\lambda$, i.e., $\left(M_{1,0}\left|C_{1}\right|-\lambda\left|C_{0}\right|\right) \times\left|C_{0}\right|=0$, and sum all equations, we have

$$
f-\lambda \sum_{J=0}^{J_{\max }}\left|C_{J}\right|^{2}=f-\lambda=0
$$

It shows that the maximum degree of orientation is the maximum value of $\lambda$ which can be obtained from Eq. (12).

Another general result is obtained by multiplying each equation in Eq. (12) by the corresponding coefficient $\left|C_{J}\right|$ for $J$ even and $-\left|C_{J}\right|$ for $J$ odd, i.e., $\left(M_{1,0}\left|C_{1}\right|-\lambda\left|C_{0}\right|\right) \times\left|C_{0}\right|=$ $0,\left(M_{1,0}\left|C_{0}\right|+M_{2,1}\left|C_{2}\right|-\lambda\left|C_{1}\right|\right) \times\left(-\left|C_{1}\right|\right)=0$, and sum all equations. If $J_{\max }$ is even, then we have

$$
\underbrace{\left(\left|C_{1}\right|^{2}+\cdots+\left|C_{J_{\max }-1}\right|^{2}\right)}_{P_{\text {odd }}}-\underbrace{\left(\left|C_{0}\right|^{2}+\cdots+\left|C_{J_{\max }}\right|^{2}\right)}_{P_{\text {even }}}=0 .
$$

It can be seen that when the maximum degree of orientation is obtained for a given $J_{\max }$, the total populations of the odd and even states are equal. If $J_{\max }$ is odd, the same conclusion can be obtained. This result has also been mentioned in Ref. [20]. It should be noted that $P_{\text {odd }}=P_{\text {even }}$ is just a necessary but
TABLE I. Molecular parameters of CO and OCS.

\begin{tabular}{llllrr}
\hline \hline & $B / \mathrm{cm}^{-1}$ & $\alpha_{\|} / \AA^{3}$ & $\alpha_{\perp} / \AA^{3}$ & \multicolumn{1}{c}{$\beta_{\|} / \AA^{5}$} & \multicolumn{1}{c}{$\beta_{\perp} / \AA^{5}$} \\
\hline CO & 1.93 & 2.294 & 1.77 & $2.748 \times 10^{9}$ & $4.994 \times 10^{8}$ \\
OCS & 0.2029 & 7.886 & 3.864 & $-3.888 \times 10^{9}$ & $-5.106 \times 10^{9}$ \\
\hline \hline
\end{tabular}

not sufficient condition for obtaining the maximum degree of orientation for a given $J_{\max }$. Thus $P_{\text {odd }}=P_{\text {even }}$ is valid for any extremum and, e.g., also obtained when all populations are equal, which is not an optimal distribution. The population distribution of the rotational states should also be considered.

The first few upper limit values of $\langle\cos \theta\rangle$ for $J_{\max }=$ $1,2,3,4$, and 5 are $0.577,0.775,0.861,0.906$, and 0.932 , respectively. It indicates that a high degree of orientation can be obtained with a broad range of the rotational quantum number $J$. We show below that the underlying assumption of constant phases is fulfilled under adiabatic conditions.

\section{RESULTS AND DISCUSSION}

The linear molecules CO and OCS are selected as models for numerical simulation. Molecular parameters are taken from Refs. [41-43] and shown in Table I. As we mainly consider the adiabatic condition during the slow turn-on of two-color laser pulses, the fall time $\sigma_{F}$ in Eq. (2) is set to zero first, corresponding to a pulse which is suddenly switched off at $t=0$. The initial rotational state is set to $J=0$.

A high degree of orientation 0.91 is obtained for $\mathrm{CO}$ with a relative long rise time 2 ns (Fig. 1). It can be seen that the adiabatic turn-on produces nearly identical phase differences $\varphi_{J, J-1}$. Further, the total populations of the odd and even states are almost equal $\left(P_{\text {odd }}=0.499\right.$ and $\left.P_{\text {even }}=0.501\right)$. That is just the necessary condition for obtaining the maximum degree of orientation for a given $J_{\max }$. The numerical result for the degree of orientation is essentially identical to the optimal

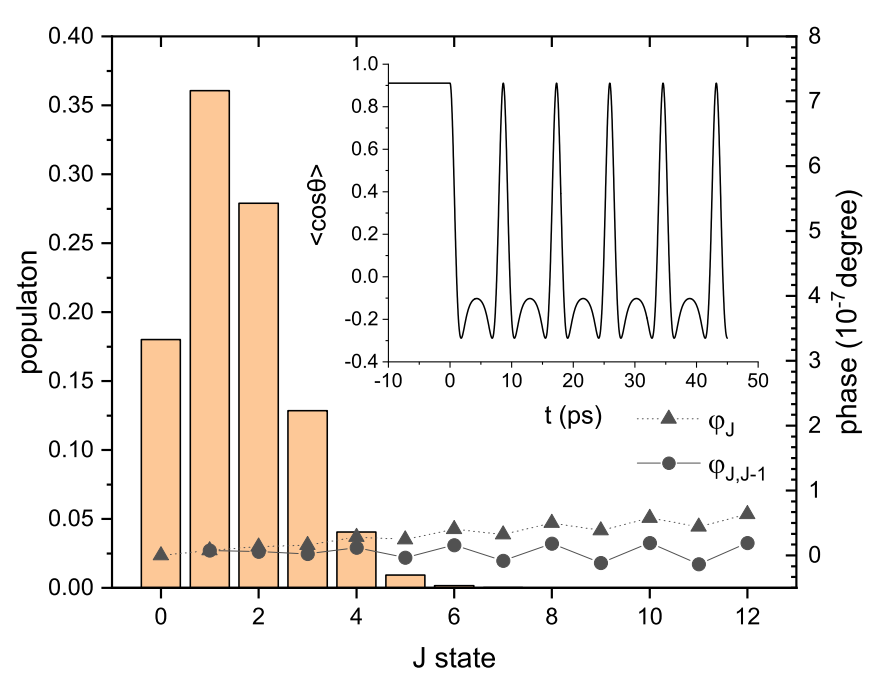

FIG. 1. Field-free population of CO, the phase of $J$ states $\varphi_{J}$ and phase differences $\varphi_{J, J-1}$ as a function of $J$. The laser parameters are $E_{0}=7 \times 10^{7} \mathrm{~V} / \mathrm{cm}, \sigma_{R}=2 \mathrm{~ns}$. The inset shows the molecular orientation after the pulse. 

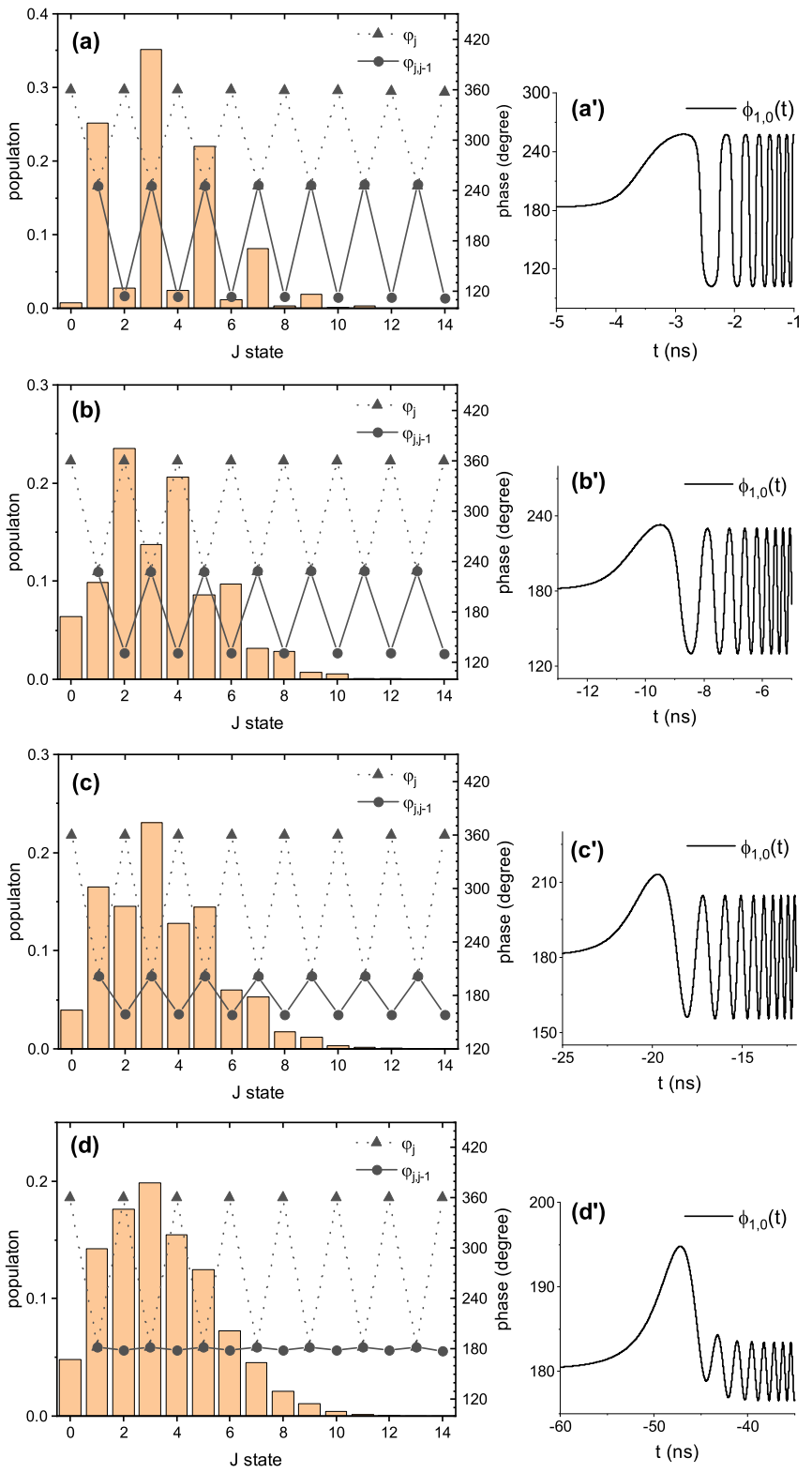

FIG. 2. Field-free population of OCS, the phase of $J$ states $\varphi_{J}$ and phase differences $\varphi_{J, J-1}$ as a function of $J$ for different rise times, (a) $\sigma_{R}=2$, (b) 6 , (c) 12 , and (d) $28 \mathrm{~ns}$. The right panels show the evolution of $\phi_{1,0}(t)$ with corresponding rise time $\left(\mathrm{a}^{\prime}\right) \sigma_{R}=2,\left(\mathrm{~b}^{\prime}\right) 6,\left(\mathrm{c}^{\prime}\right)$ 12 , and $\left(\mathrm{d}^{\prime}\right) 28 \mathrm{~ns}$. Peak electric amplitude $E_{0}=2.745 \times 10^{7} \mathrm{~V} / \mathrm{cm}$.

result for $J_{\max }=5$. The population distribution is similar to but is not identical with the analytically obtained result.

While under the same rise time, it is difficult to get identical phases for OCS [Fig. 2(a)], we found that the odd and even rotational states are in phase, respectively. One way to get the same phase difference $\varphi_{J, J-1}$ between $J$ and $J-1$ states is by further increasing the rise time to obtain an adiabatic evolution of the molecular states (Fig. 2). The phase difference $\varphi_{J, J-1}$ approaches nearly the same value $\pi$ after a long rise time of $28 \mathrm{~ns}$ [Fig. 2(d)].

From the evolution of the phase difference $\phi_{1,0}(t)$ [Figs. 2( $\left.a^{\prime}\right)-2\left(d^{\prime}\right)$ ], we find a "bump" (the first sudden phase difference change) which decreases as the rise time increases.
After the bump, $\phi_{1,0}(t)$ begins to oscillate with a specific amplitude. This kind of oscillation implies that the phase difference $\varphi_{j, j-1}$ cannot be kept constant. It can be used as a signature to justify if the adiabatic condition is satisfied by observing the evolution of the phase difference between adjacent rotational states over time.

Furthermore, the total populations of the odd and even states are very different for the case of $\sigma_{R}=2 \mathrm{~ns}$ [Fig. 2(a)], but become nearly equal as the rise time increase. For the case of $\sigma_{R}=28$ ns [Fig. 2(d)], $P_{\text {odd }}=0.523$ and $P_{\text {even }}=0.477$. The dependence of the total populations of the odd and even states on the peak amplitude of the electric field $E_{0}$ for OCS with $\sigma_{R}=28 \mathrm{~ns}$ is shown in Table II. Only even rotational states are populated when $E_{0}$ is weak. As the $E_{0}$ increases, $P_{\text {odd }}$ and $P_{\text {even }}$ approach 0.5 . When $E_{0}$ exceeds a certain value, the difference between $P_{\text {odd }}$ and $P_{\text {even }}$ starts to increase. This is due to the effect of electric field intensity on the adiabatic dynamics, which will be shown in Sec. III A 1. It can be seen that $P_{\text {odd }}=P_{\text {even }}$ can be obtained at a sufficiently high intensity with adiabatic dynamics.

The degree of orientation in Fig. 2(d) reaches 0.973 . The numerical result for the degree of orientation is essentially identical to the optimal result for $J_{\max }=10\left(\langle\cos \theta\rangle_{\max }=\right.$ 0.978). It indicates that a nearly optimal distribution of rotational states can be obtained at a sufficiently high intensity with adiabatic dynamics. Again, the population distribution is similar to but is not identical with the analytically obtained result, indicating that the optimal degree of orientation is not highly sensitive to the population distribution. Ionization is negligible at the applied laser intensity [35]. It should be noted that the peak amplitude $E_{0}$ determines the range of the rotational quantum number $J$ for a specific molecule in our two-color truncated pulses scheme. As discussed in Sec. II B, $J_{\max }$ determines the upper limit value of the maximum degree of orientation, and the optimal distribution of rotational states varies for different $J_{\max }$. In order to get a higher degree of orientation, increasing the peak amplitude $E_{0}$ should be considered first, and then the adiabatic dynamics should be ensured.

As the adiabatic process is beneficial for getting a high degree of orientation, it is crucial to understand what influences the adiabatic process. Based on the adiabatic theorem [Eq. (6)], one of the important factors is the coupling matrix element which is related to the rate of change of the Hamiltonian and the other one is the instantaneous eigenenergy difference. There are several parameters affecting the rate of change of the Hamiltonian [Eq. (7)]; anisotropic polarizability $\Delta \alpha$, hyperpolarizability $\beta_{\|}, \beta_{\perp}$, peak amplitude $E_{0}$, and rise time $\sigma_{R}$. The instantaneous eigenenergy difference is determined by $\hat{H}(t)$. Comparing to the alignment case, near degeneracy of states in the double-well potential is removed in the two-color laser pulse scheme. The dependence of the double-well potential on the parameters should be considered.

On the other hand, assuming that the molecule evolves from a certain initial state and goes through an adiabatic process, the maximum degree of orientation is determined by the instantaneous state at $t=0$, which is dependent on parameters of the laser and the molecule. This will guide us how to optimize the laser or choose the molecule in order to obtain a high degree of molecular orientation. The influence of 
TABLE II. The dependence of $P_{\text {odd }}$ and $P_{\text {even }}$ on the peak amplitude of electric field $E_{0}$ for OCS with $\sigma_{R}=28$ ns. The range of the laser intensities is $4.8 \times 10^{10}-1 \times 10^{12} \mathrm{~W} / \mathrm{cm}^{2}$.

\begin{tabular}{lcccccccrrr}
\hline \hline$E_{0}\left(10^{7} \mathrm{~V} / \mathrm{cm}\right)$ & 0.6 & 0.65 & 0.68 & 0.71 & 0.74 & 0.78 & 1 & 1.5 & 2 \\
\hline$P_{\text {odd }}$ & 0.039 & 0.132 & 0.220 & 0.306 & 0.373 & 0.430 & 0.499 & 0.495 & 0.518 & 0.523 \\
$P_{\text {even }}$ & 0.961 & 0.868 & 0.780 & 0.694 & 0.627 & 0.570 & 0.501 & 0.505 & 0.482 & 0.477 \\
\hline \hline
\end{tabular}

parameters will be discussed by considering both the adiabatic process and the degree of molecular orientation.

\section{A. Influence of laser parameters \\ 1. Peak amplitude $E_{0}$}

Figure 3 shows the time dependence of the coupling matrix element $|\langle\tilde{0}, 0|\partial \hat{H} / \partial t| \tilde{1}, 0\rangle|$ and instantaneous energy difference of the two lowest pendular states, $|\tilde{0}, 0\rangle$ and $|\tilde{1}, 0\rangle$, with a constant $\sigma_{R}$ and different peak amplitudes. A maximum of $|\langle\tilde{0}, 0|\partial \hat{H} / \partial t| \tilde{1}, 0\rangle|$ and a minimum of $\left|\left(E_{n}-E_{m}\right)^{2} / \hbar\right|$ appears roughly at the same time during the pulse. If there is a big gap between the two lines, the adiabatic condition is well fulfilled and the bump and amplitude of the phase oscillation in Fig. 2 will get smaller. As the peak intensity decreases, the gap increases, which makes it easier to meet the adiabatic condition. That can be explained from Eq. (7), a smaller $E_{0}$ reduces the rate of change of the Hamiltonian.

However, it should be noted that the maximum of $|\langle\tilde{0}, 0|\partial \hat{H} / \partial t| \tilde{1}, 0\rangle|$ and $\partial \hat{H} / \partial t$ mostly occurs at different times. The former occurs at roughly the same time where the minimum of the instantaneous energy difference occurs, and the latter is easy to get from Eq. (7). The instantaneous eigenvalues are related to the shape of the double-well potential which changes with electric field intensity $\varepsilon(t)$. We observe, for different $E_{0}$, the minimum values of the instantaneous eigenvalue difference between $E_{\tilde{1}, 0}$ and $E_{\tilde{0}, 0}$ are the same for a specific molecule. That means once the electric field

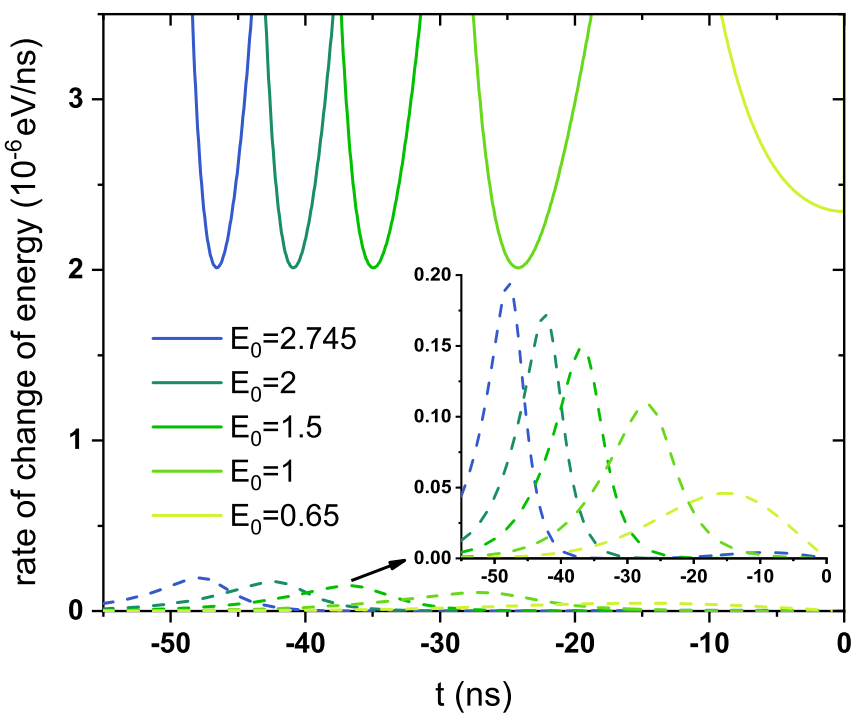

FIG. 3. Time dependence of $\left|\left(E_{\tilde{1}, 0}-E_{\tilde{0}, 0}\right)^{2} / \hbar\right|$ (solid lines) and $|\langle\tilde{0}, 0|\partial \hat{H} / \partial t| \tilde{1}, 0\rangle|$ (dashed lines) for OCS for different electric field peak amplitudes $E_{0}$ in unit of $10^{7} \mathrm{~V} / \mathrm{cm}$. The rise time $\sigma_{R}$ is set to $28 \mathrm{~ns}$. The inset is an enlarged image of the dashed lines. intensity reaches a certain value $\varepsilon_{c}$ during the slow turn-on of the pulse, the minimum of $\left|\left(E_{\tilde{1}, 0}-E_{\tilde{0}, 0}\right)^{2} / \hbar\right|$ will be obtained except when the peak amplitude $E_{0}$ is smaller than $\varepsilon_{c}$. At the time where the minimum of $\left|\left(E_{\tilde{1}, 0}-E_{\tilde{0}, 0}\right)^{2} / \hbar\right|$ occurs, we find that $\varepsilon_{c}=0.688 \times 10^{7} \mathrm{~V} / \mathrm{cm}$ for OCS. For the case of $E_{0}=0.65 \times 10^{7} \mathrm{~V} / \mathrm{cm}$ (the inset in Fig. 3), the maximum of $|\langle\tilde{0}, 0|\partial \hat{H} / \partial t| \tilde{1}, 0\rangle|$ and $\partial \hat{H} / \partial t$ occurs at almost the same time. $\varepsilon_{c}$ can be substituted into Eq. (7) with the time $t$ given by Eq. (2), then we have

$$
\begin{aligned}
\left.\frac{\partial \hat{H}}{\partial t}\right|_{\varepsilon(t)=\varepsilon_{c}}= & -\Delta \alpha \cos ^{2} \theta \varepsilon_{c}^{2} \frac{\sqrt{2 \ln \left(E_{0} / \varepsilon_{c}\right)}}{\sigma_{R}} \\
& -\frac{3}{8}\left[\left(\beta_{\|}-3 \beta_{\perp}\right) \cos ^{3} \theta+3 \beta_{\perp} \cos \theta\right] \\
& \times \varepsilon_{c}^{3} \frac{\sqrt{2 \ln \left(E_{0} / \varepsilon_{c}\right)}}{\sigma_{R}} .
\end{aligned}
$$

This equation gives the maximum value of the time derivative of the Hamiltonian during the turn-on of the pulse. There will be nonadiabatic transitions if this value is too large as we increase the peak amplitude for a given $\sigma_{R}$. After this maximum, the adiabatic condition becomes satisfied again.

Although adiabatic conditions are obtained with a smaller peak amplitude $E_{0}$, it is not equivalent to obtaining a high degree of molecular orientation. The wave function of the ground states $|\tilde{0}, 0\rangle$ with different peak amplitudes at $t=0$ are compared in Fig. 4. Higher peak amplitude results in a deeper and more asymmetric double-well potential. A deeper asymmetric double-well potential will make the peak of the wave function of ground states $|\tilde{0}, 0\rangle$ higher and narrower which is beneficial for getting a higher degree of orientation. For the case of $E_{0}=0.65 \times 10^{7} \mathrm{~V} / \mathrm{cm}$, the wave function of the ground state has not yet been transformed into a singlepeak shape. The degree of orientation at $t=0$ as a function of $E_{0}$ is shown in the inset of Fig. 4. The calculated results based on the wave function of the ground states $|\tilde{0}, 0\rangle$ or all states are almost the same. It indicates that the dynamics is adiabatic and the wave function of the ground state $|\tilde{0}, 0\rangle$ can be used to calculate the degree of orientation. It can be seen that the degree of orientation is sensitive to $E_{0}$, at first where the wave function of $|\tilde{0}, 0\rangle$ has a double-peak shape and then the slope gets small when a single-peak shape wave function is formed. Thus, a relative high intensity is needed to get a good molecular orientation, equivalent to a sufficiently high value of $J_{\max }$ in Eq. (9).

\section{Rise time $\sigma_{R}$}

In order to obtain a high degree of orientation at high peak intensity and keep the adiabatic condition at the same time, another key parameter, the rise time $\sigma_{R}$ should be taken into account. It can be seen from Eq. (15) that $\partial \hat{H} / \partial t$, at the 


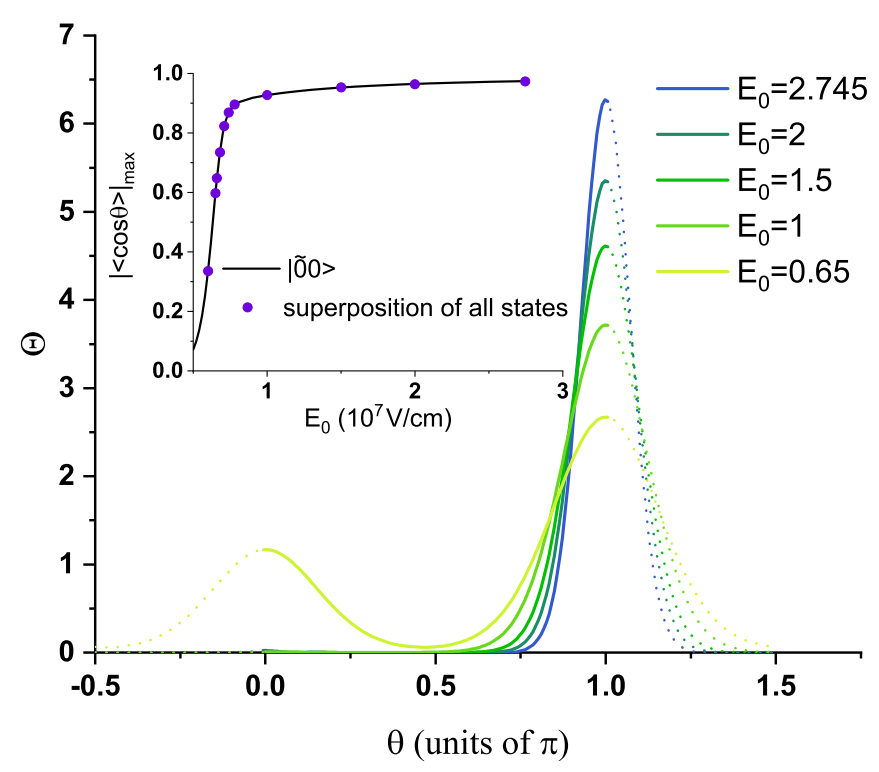

FIG. 4. The wave function of the instantaneous ground states $|\tilde{0}, 0\rangle$ of OCS at $t=0$ corresponding to different peak amplitudes $E_{0}$ in the unit of $10^{7} \mathrm{~V} / \mathrm{cm}$. The rise time $\sigma_{R}$ is set to $28 \mathrm{~ns}$. The solid line is the normalized $\Theta(\theta)$ of the spherical harmonics. The dotted line represents the periodic extension for showing the wave function in a double-well potential more clearly. The inset shows the degree of orientation at $t=0$ as a function of $E_{0}$. The solid line is obtained from the wave function of $|\tilde{0}, 0\rangle$. The circles are obtained from the superposition of all the pendular states.

moment of $\varepsilon(t)=\varepsilon_{c}$, is inversely proportional to $\sigma_{R}$. Then we can increase $\sigma_{R}$ to reduce the maximum rate of change of the Hamiltonian until the adiabatic condition is satisfied (Fig. 5). As the shape of double-well potential at $t=0$ is independent of the rise time $\sigma_{R}$, it is an effective method to increase the

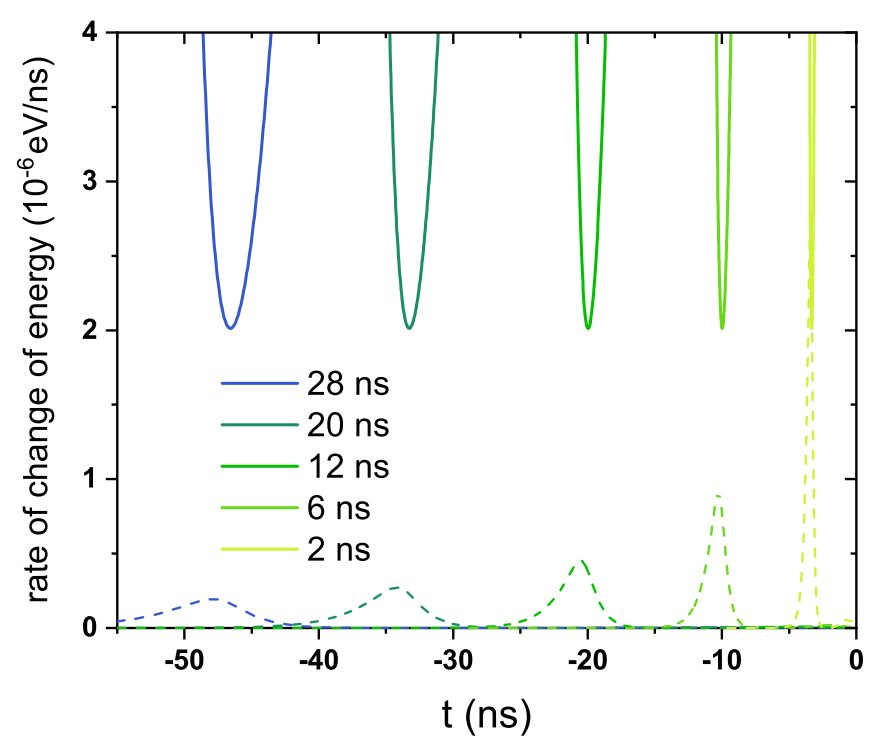

FIG. 5. Time dependence of $\left|\left(E_{\tilde{1}, 0}-E_{\tilde{0}, 0}\right)^{2} / \hbar\right|$ (solid lines) and $|\langle\tilde{0}, 0|\partial \hat{H} / \partial t| \tilde{1}, 0\rangle|$ (dashed lines) for OCS with different laser rise times $\sigma_{R}$. The electric field peak amplitude $E_{0}$ is set to $2.745 \times 10^{7} \mathrm{~V} / \mathrm{cm}$. rise time to get an adiabatic process and a high degree of orientation.

Comparing Fig. 5 to the evolution of the phase difference in Fig. 2, for the same $\sigma_{R}$, the "bump" just occurs at the moment of $\varepsilon(t)=\varepsilon_{c}$ where the nonadiabatic transition is most likely to appear. It shows clearly that the sudden change in the phase difference is caused by the nonadiabatic process. For the case of $\sigma_{R}=28$ ns in Fig. 5, $\left|\left(E_{\tilde{1}, 0}-E_{\tilde{0}, 0}\right)^{2} / \hbar\right|$ is one order of magnitude larger than $|\langle\tilde{0}, 0|\partial \hat{H} / \partial t| \tilde{1}, 0\rangle|$ and the amplitude of the phase difference oscillation is about $6^{\circ}$ [Fig. $\left.2\left(\mathrm{~d}^{\prime}\right)\right]$. This leads to the variation of $\varphi_{J, J-1}$ [Fig. 2(d)], which is much larger than that obtained for $\mathrm{CO}$ (Fig. 1). Further, comparing to the case of $\mathrm{CO}$, the difference between $P_{\text {odd }}$ and $P_{\text {even }}$ is larger for OCS in Fig. 2(d). Although the adiabatic process is better satisfied and the peak intensity of the laser is higher, the $J_{\max }$ in the case of $\mathrm{CO}$ (Fig. 1) is smaller than that of OCS [Fig. 2(d)], and CO does not obtain a higher degree of orientation than OCS. That means that the choice of molecules is also very important.

\section{B. Influence of molecular parameters}

The Hamiltonian depends not only on the parameters of the laser but also on the molecular parameters. The choice of molecule determines how easy it is to meet the adiabatic criterion. Here we keep the parameters of the laser constant and change $B, \Delta \alpha, \beta_{\|}$and $\beta_{\perp}$, respectively, to investigate the adiabatic process and the dependence of the degree of orientation on molecular parameters.

As shown in Fig. 6(a), the minimum of $\left|\left(E_{\tilde{1}, 0}-E_{\tilde{0}, 0}\right)^{2} / \hbar\right|$ increases with the increase of the rotational constant $B$ or with the decrease of $\Delta \alpha$. The same trend can be also found in the change of the maximum of $|\langle\tilde{0}, 0|\partial \hat{H} / \partial t| \tilde{1}, 0\rangle|$ over time. The change of the former is several orders of magnitude larger than that of the latter [inset of Fig. 6(a)]. Thus molecules with larger $B$ and smaller $\Delta \alpha$ are beneficial for achieving an adiabatic process. On the other hand, a smaller $B$ and larger $\Delta \alpha$ results in a more localized wave function [inset of Fig. 6(b)]. That is why a higher degree of orientation is obtained for OCS at lower peak intensity, even when there is a bit of nonadiabatic effects during the evolution compared to the case of $\mathrm{CO}$.

Further, it can be found from Figs. 6(a) and 6(b) that, after the instantaneous eigenvalue difference reaches its minimum, the curves with the same value of $\Delta \alpha / B$ begin to approach and coincide, with the increase of laser intensity. The instantaneous ground-state eigenfunctions at $t=0$ are essentially identical for the same value of $\Delta \alpha / B$ [inset of Fig. 6(b)]. In addition, we observed that when the value of $\Delta \alpha / B$ is kept constant, the instantaneous eigenfunctions at $t=0$ (where the intensity is sufficiently high) are almost independent of the molecular hyperpolarizabilities. It will be explained in Sec. III C.

Molecular hyperpolarizability affects the minimum value of the eigenvalue difference (Fig. 7). Larger absolute values of the hyperpolarizability, which makes the double-well potential more asymmetric, increase both the minimum of $\left|\left(E_{\tilde{1}, 0}-E_{\tilde{0}, 0}\right)^{2} / \hbar\right|$ and the maximum of $|\langle\tilde{0}, 0|\partial \hat{H} / \partial t| \tilde{1}, 0\rangle|$, but by different degrees just like the case of $B$ and $\Delta \alpha$. Thus, larger absolute values of the hyperpolarizability are beneficial 

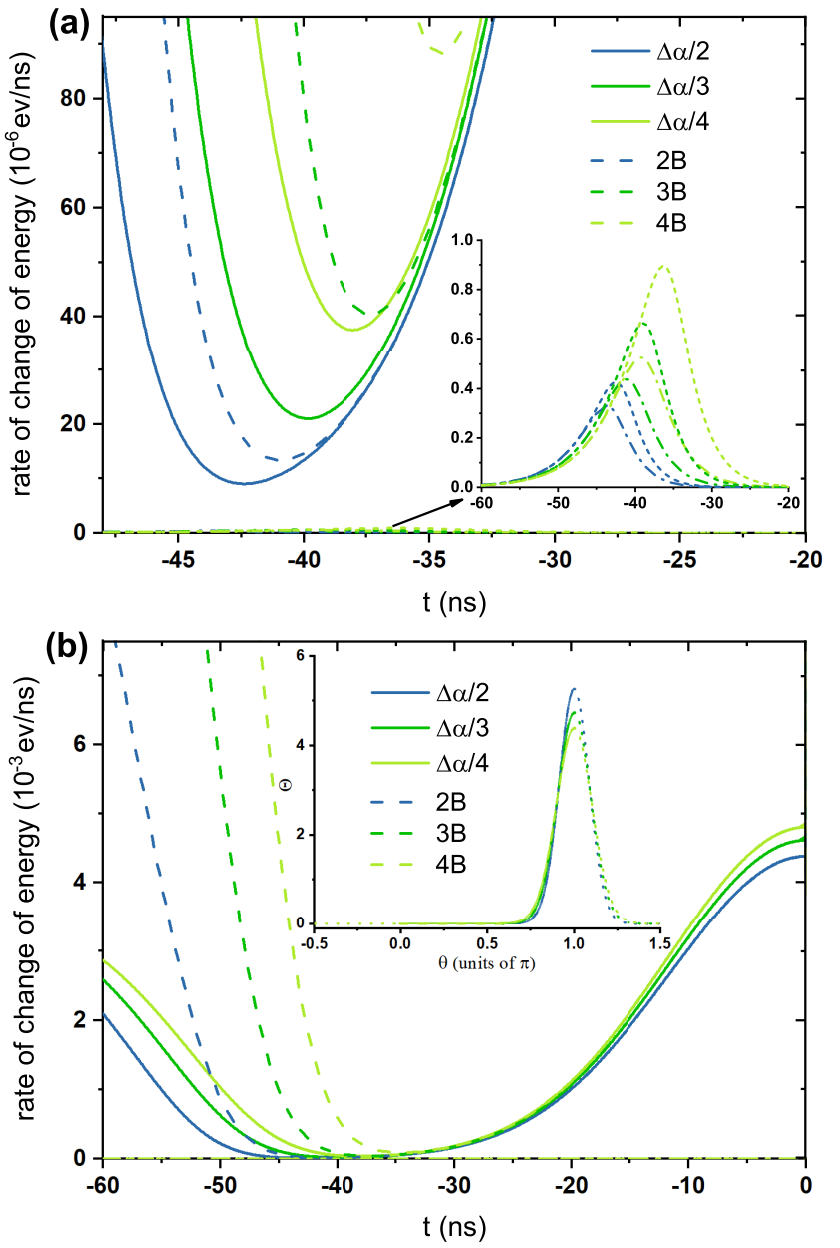

FIG. 6. (a) Time dependence of $\left|\left(E_{\tilde{1}, 0}-E_{\tilde{0}, 0}\right)^{2} / \hbar\right|$ (solid and dashed lines) and $|\langle\tilde{0}, 0|\partial \hat{H} / \partial t| \tilde{1}, 0\rangle|$ (dashed-dot and short-dashed lines) for a linear molecule with different rotational constants and anisotropic polarizability. $B, \Delta \alpha, \beta_{\|}$, and $\beta_{\perp}$ are the parameters of the OCS molecule. The electric field peak amplitude and rise time are kept constant $E_{0}=2.745 \times 10^{7} \mathrm{~V} / \mathrm{cm}$ and $\sigma_{R}=28 \mathrm{~ns}$. The inset is an enlarged image of the dashed lines. (b) is the identical image of (a) with larger rate of change of energy and time scales. The inset shows the wave function of the instantaneous ground states of OCS at $t=0$ with different parameters represented by corresponding colors. The solid and dashed lines are the normalized $\Theta(\theta)$ of the spherical harmonics. The dotted line represents the periodic extension for showing the wave function in a double-well potential more clearly.

for achieving an adiabatic process and hardly affects the peak of the wave function when the intensity is high enough.

Although it is difficult to achieve an adiabatic process for linear molecules with a larger $\Delta \alpha / B$, it is beneficial for projecting the pendular states created during the turn-on of the laser pulse onto free-rotor state. The case in Fig. 2(d) for OCS and Fig. 1 for CO are compared to analyze the effect of the fall time on the degree of molecular orientation. It can be seen from Fig. 8 that the maximum degree of orientation from an adiabatic process for OCS is less sensitive to the fall time than that for $\mathrm{CO}$. As the fall time $\sigma_{F}$ increases to $2 \mathrm{ps}$, the maximum degree of orientation for CO decreases by $28 \%$, while that for OCS only decreases by $4.2 \%$.
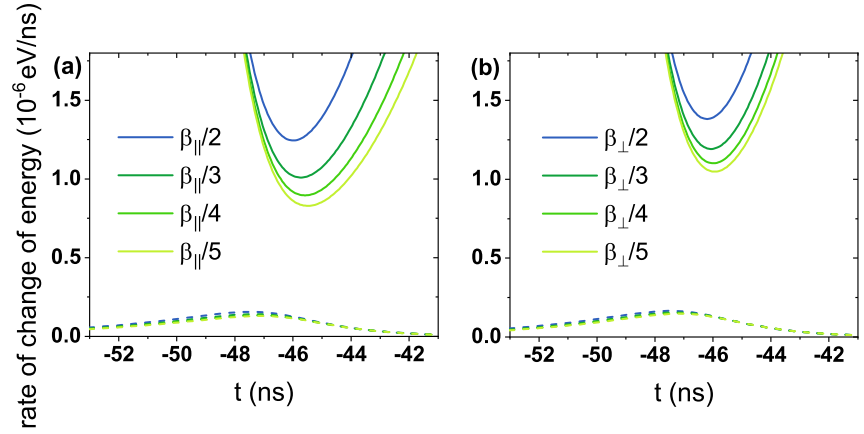

FIG. 7. Time dependence of $\left|\left(E_{\tilde{1}, 0}-E_{\tilde{0}, 0}\right)^{2} / \hbar\right|$ (solid lines) and $|\langle\tilde{0}, 0|\partial \hat{H} / \partial t| \tilde{1}, 0\rangle|$ (dashed lines) for a linear molecule with different hyperpolarizabilities (a) parallel and (b) perpendicular to the molecular axis. $B, \Delta \alpha, \beta_{\|}$, and $\beta_{\perp}$ are the parameters of the OCS molecule. The electric field peak amplitude and rise time are kept constant $E_{0}=2.745 \times 10^{7} \mathrm{~V} / \mathrm{cm}$ and $\sigma_{R}=28 \mathrm{~ns}$.

\section{Theoretical analysis in a two-state model}

The analysis of the previous section is now supplemented by an analytical model. The eigenfunctions in a symmetric double-well potential occur as tunneling doublets with opposite parity. In our calculations, the polarizability term is several orders larger than the hyperpolarizability term and the hyperpolarizability term which breaks the symmetry can be taken as a perturbation. Thus, we use a two-state model, which has been used to analyze the mixed-field scheme [25], to explain some of our previous results.

The Hamiltonian [Eq. (3)] can be separated into two parts, $\hat{H}=\hat{H}_{0}+\hat{H}^{\prime}$, where

$$
\begin{gathered}
\hat{H}_{0}(t)=B \hat{J}^{2}-\frac{\varepsilon^{2}(t)}{2} \Delta \alpha \cos ^{2} \theta, \\
\hat{H}^{\prime}(t)=-\frac{\varepsilon^{3}(t)}{8}\left[\left(\beta_{\|}-3 \beta_{\perp}\right) \cos ^{3} \theta+3 \beta_{\perp} \cos \theta\right] .
\end{gathered}
$$

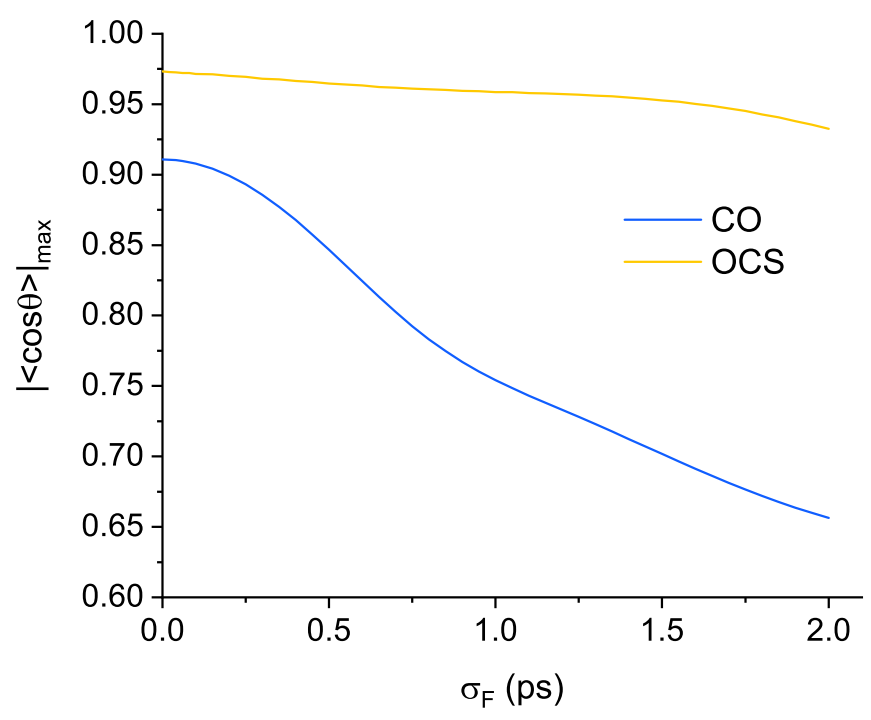

FIG. 8. Fall time $\sigma_{F}$ dependence of the maximum degree of orientation for $\mathrm{CO}$ and OCS. The peak amplitude and rise time of the laser pulse for $\mathrm{CO}$ are identical to the parameters in Fig. 1 $\left(E_{0}=7 \times 10^{7} \mathrm{~V} / \mathrm{cm}\right.$ and $\left.\sigma_{R}=2 \mathrm{~ns}\right)$ and that for OCS are identical to the parameters in Fig. $2(\mathrm{~d})\left(E_{0}=2.745 \times 10^{7} \mathrm{~V} / \mathrm{cm}\right.$ and $\left.\sigma_{R}=28 \mathrm{~ns}\right)$. 


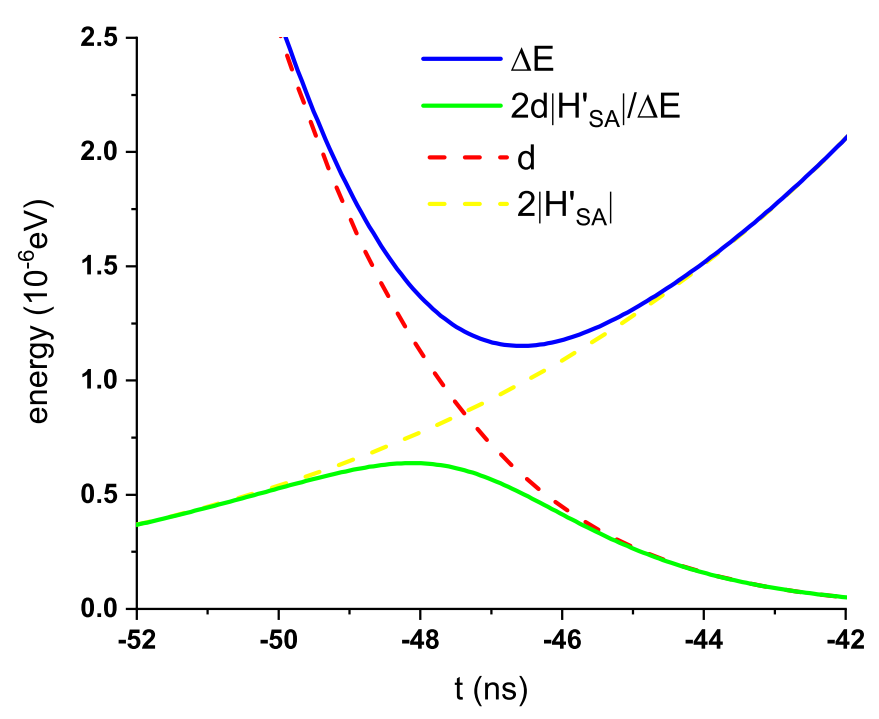

FIG. 9. Schematic diagram of the time dependence of $d, 2\left|H_{S A}^{\prime}\right|$, $\Delta E$, and $2 d\left|H_{S A}^{\prime}\right| / \Delta E$. OCS is considered as a model. The electric field peak amplitude and rise time are $E_{0}=2.745 \times 10^{7} \mathrm{~V} / \mathrm{cm}$ and $\sigma_{R}=28 \mathrm{~ns}$, respectively.

The energy splitting of the doublets in $\hat{H}_{0}$ can be expressed as $d=E_{A}-E_{S}$, where $E_{S}$ and $E_{A}$ are instantaneous eigenvalues of $\hat{H}_{0}$. The corresponding eigenfunctions are denoted as $\left|\psi_{S}\right\rangle$ and $\left|\psi_{A}\right\rangle$ which have opposite parity. Further, $\left|\psi_{S}\right\rangle$ only have even numbers of $J$ and $\left|\psi_{A}\right\rangle$ only have odd numbers of $J$ in the $|J, M\rangle$ representation [25]. In this two-state model $\hat{H}$ can be expressed as

$$
\left[\begin{array}{cc}
E_{S} & H_{S A}^{\prime} \\
H_{A S}^{\prime} & E_{A}
\end{array}\right],
$$

where $H_{S A}^{\prime}=\left\langle\psi_{S}\left|\hat{H}^{\prime}\right| \psi_{A}\right\rangle=H_{A S}^{\prime *}=H_{A S}^{\prime}$. Then, the exact eigenvalues and eigenfunctions of $\hat{H}$ are

$$
E_{ \pm}=\frac{E_{A}+E_{S}}{2} \pm \frac{1}{2} \sqrt{d^{2}+4 H_{S A}^{\prime 2}}
$$

and

$$
\begin{aligned}
& \left|\psi_{+}\right\rangle=\sin \chi\left|\psi_{S}\right\rangle+\cos \chi\left|\psi_{A}\right\rangle, \\
& \left|\psi_{-}\right\rangle=\cos \chi\left|\psi_{S}\right\rangle-\sin \chi\left|\psi_{A}\right\rangle
\end{aligned}
$$

with the mixing angle determined by

$$
\tan (2 \chi)=\frac{2 H_{S A}^{\prime}}{d}
$$

The eigenvalue difference can be written as

$$
\Delta E=E_{+}-E_{-}=\sqrt{d^{2}+4 H_{S A}^{\prime 2}} .
$$

It can be seen from Fig. 9 that $\left|H_{S A}^{\prime}\right|$ increases monotonically and $d$ decreases monotonically as $\varepsilon(t)$ increases with $t$. Meanwhile, $d \gg 2\left|H_{S A}^{\prime}\right|$ and $d \ll 2\left|H_{S A}^{\prime}\right|$ in the earlier and later period of the turn-on of the laser pulse, respectively. Therefore $\Delta E$ has a minimum which appears around the time when $d=2\left|H_{S A}^{\prime}\right|$.

Furthermore, it can be explained why the maximum of the coupling matrix element $|\langle m|\partial \hat{H} / \partial t| n\rangle|$ in Eq. (6) appears around the time when the eigenvalue difference reaches its minimum; but not the time when $\partial \hat{H} / \partial t$ is at its maximum. The coupling matrix element of $\partial \hat{H} / \partial t$ in this two-state model can be expressed as

$$
\begin{aligned}
\left\langle\psi_{-}\left|\frac{\partial \hat{H}}{\partial t}\right| \psi_{+}\right\rangle= & \left\langle\psi_{-}\left|\frac{\partial \hat{H}_{0}}{\partial t}\right| \psi_{+}\right\rangle+\left\langle\psi_{-}\left|\frac{\partial \hat{H}^{\prime}}{\partial t}\right| \psi_{+}\right\rangle \\
= & \left(\left\langle\psi_{-}\left|B \hat{J}^{2}\right| \psi_{+}\right\rangle-\left\langle\psi_{-}\left|\hat{H}_{0}\right| \psi_{+}\right\rangle\right) \frac{2 t}{\sigma^{2}} \\
& -\left\langle\psi_{-}\left|\hat{H}^{\prime}\right| \psi_{+}\right\rangle \frac{3 t}{\sigma^{2}},
\end{aligned}
$$

where

$$
\begin{aligned}
\left\langle\psi_{-}\left|B \hat{J}^{2}\right| \psi_{+}\right\rangle= & \cos \chi \sin \chi\left\langle\psi_{S}\left|B \hat{J}^{2}\right| \psi_{S}\right\rangle-\sin ^{2} \chi\left\langle\psi_{A}\left|B \hat{J}^{2}\right| \psi_{S}\right\rangle \\
& +\cos ^{2} \chi\left\langle\psi_{S}\left|B \hat{J}^{2}\right| \psi_{A}\right\rangle-\cos \chi \sin \chi\left\langle\psi_{A}\left|B \hat{J}^{2}\right| \psi_{A}\right\rangle \\
= & \cos \chi \sin \chi\left(\left\langle\psi_{S}\left|B \hat{J}^{2}\right| \psi_{S}\right\rangle-\left\langle\psi_{A}\left|B \hat{J}^{2}\right| \psi_{A}\right\rangle\right) \\
= & \frac{1}{2} \sin 2 \chi\left(\left\langle\psi_{S}\left|B \hat{J}^{2}\right| \psi_{S}\right\rangle-\left\langle\psi_{A}\left|B \hat{J}^{2}\right| \psi_{A}\right\rangle\right)
\end{aligned}
$$

and

$$
\begin{aligned}
& \left\langle\psi_{-}\left|\hat{H}_{0}\right| \psi_{+}\right\rangle=\left(E_{S}-E_{A}\right) \cos \chi \sin \chi=-\frac{d}{2} \sin 2 \chi, \\
& \left\langle\psi_{-}\left|\hat{H}^{\prime}\right| \psi_{+}\right\rangle=\left(\cos ^{2} \chi-\sin ^{2} \chi\right) H_{S A}^{\prime}=\cos 2 \chi H_{S A}^{\prime} .
\end{aligned}
$$

Substitute Eqs. (21) and (24)-(26) into Eq. (23), then we have

$$
\begin{aligned}
\left|\left\langle\psi_{-}\left|\frac{\partial \hat{H}}{\partial t}\right| \psi_{+}\right\rangle\right| & =\left|(-\Delta K+d) \sin 2 \chi \frac{t}{\sigma^{2}}-\cos 2 \chi H_{S A}^{\prime} \frac{3 t}{\sigma^{2}}\right| \\
& =\frac{2 d\left|H_{S A}^{\prime}\right|}{\Delta E}\left|\frac{t}{\sigma^{2}}\left(\frac{1}{2}+\frac{\Delta K}{d}\right)\right|
\end{aligned}
$$

where the kinetic energy difference can be written as $\Delta K=$ $\left\langle\psi_{A}\left|B \hat{J}^{2}\right| \psi_{A}\right\rangle-\left\langle\psi_{S}\left|B \hat{J}^{2}\right| \psi_{S}\right\rangle$.

As shown in Fig. 9, $2 d\left|H_{S A}^{\prime}\right| / \Delta E \approx 2\left|H_{S A}^{\prime}\right|$ and $2 d\left|H_{S A}^{\prime}\right| / \Delta E \approx d$ in the earlier and later period of the turn-on of the laser pulse, respectively. That is why the maximum of $2 d\left|H_{S A}^{\prime}\right| / \Delta E$ always appears around the time when $d=2\left|H_{S A}^{\prime}\right|$. The maximum is shifted when $2 d\left|H_{S A}^{\prime}\right| / \Delta E$ is multiplied by the term including $t$.

We can roughly evaluate if the adiabatic condition is fulfilled by inserting Eq. (22) and Eq. (27) in Eq. (6) when $d=2\left|H_{S A}^{\prime}\right|$,

$$
\begin{aligned}
\left|\hbar\left\langle\psi_{-}\left|\frac{\partial \hat{H}}{\partial t}\right| \psi_{+}\right\rangle / \Delta E^{2}\right| & =\frac{\hbar}{2 \sqrt{2} d}\left|\frac{t}{\sigma^{2}}\left(\frac{1}{2}+\frac{\Delta K}{d}\right)\right| \\
& =\frac{\hbar}{4 \sqrt{2}\left|H_{S A}^{\prime}\right|}\left|\frac{t}{\sigma^{2}}\left(\frac{1}{2}+\frac{\Delta K}{d}\right)\right| .
\end{aligned}
$$

Considering that $d$ increases as $B$ increases or as $\Delta \alpha$ decreases [25] and that $\left|H_{S A}^{\prime}\right|$ is proportional to $\left|\beta_{\|}\right|$or $\left|\beta_{\perp}\right|$, a molecule with a large $B,\left|\beta_{\|}\right|$, or $\left|\beta_{\perp}\right|$ and a small $\Delta \alpha$ can easily fulfill the adiabatic condition which is consistent with the results in Sec. III B.

Since $H_{S A}^{\prime}=0$ before the turn-on of the laser pulse and $d$ approaches 0 at a high electric field, the mixing angle $2 \chi$ in Eq. (21) changes from 0 to $\pi / 2$ or $-\pi / 2$. Thus, as the intensity gets sufficiently strong, $\chi$ approaches $\pi / 4$ or $-\pi / 4$. 
Then, the eigenfunctions of $\hat{H}$ in Eq. (20) can be written as

$$
\begin{aligned}
\left|\psi_{ \pm}\right\rangle & =\frac{1}{\sqrt{2}}\left(\left|\psi_{S}\right\rangle \pm\left|\psi_{A}\right\rangle\right) \\
\text { or }\left|\psi_{ \pm}\right\rangle & =\frac{1}{\sqrt{2}}\left(\left|\psi_{A}\right\rangle \mp\left|\psi_{S}\right\rangle\right),
\end{aligned}
$$

where $\left|\psi_{S}\right\rangle$ and $\left|\psi_{A}\right\rangle$ are determined by $\hat{H}_{0}$ [Eq. (16)]. That is why the instantaneous eigenfunction is independent of the molecular hyperpolarizability at a sufficiently high laser intensity.

According to the expression for $\hat{H}_{0}$ [Eq. (16)], after dividing both sides of the Schrödinger equation $\hat{H}_{0}(t) \psi=E(t) \psi$ by $B$, it can be found that the depth of the double-well potential is proportional to $\Delta \alpha / B$ for any linear molecule. As $\Delta \alpha / B$ increases, the peak of $\left|\psi_{S}\right\rangle$ and $\left|\psi_{A}\right\rangle$ increase. That is why a "sharp" wave function can be obtained with a larger $\Delta \alpha / B$ under the superposition of $\left|\psi_{S}\right\rangle$ and $\left|\psi_{A}\right\rangle$ which are of opposite parity (Eq. (29)). Furthermore, if the dynamics is adiabatic, the system evolves into $\left|\psi_{+}\right\rangle$or $\left|\psi_{-}\right\rangle$. As $\left|\psi_{S}\right\rangle$ only have even numbers of $J$ and $\left|\psi_{A}\right\rangle$ only have odd numbers of $J$ in the $|J, M\rangle$ representation, it can be seen from Eq. (29) that identical total populations of the odd and even states can be obtained at a sufficiently high electric field with adiabatic dynamics.

\section{SUMMARY}

We investigated the effects of the parameters of twocolor slow turn-on and rapid turn-off laser pulses for linear molecules, respectively, on the adiabatic condition and the degree of molecular orientation. Analytical results for the maximum degree of field-free orientation for a given number of populated rotational states is derived. The adiabatic dynamics turns out to be a very effective method to get a high degree of field-free orientation, by producing identical phase differences of adjacent rotational states, identical total populations of the odd and even states, and a nearly optimal distribution of rotational states at a sufficiently high peak intensity of the laser.

In order to obtain a high degree of orientation, a high electric field amplitude is required which makes it difficult to obtain an adiabatic process. However, we can increase the rise time of the laser pulse to obtain an adiabatic process for a high peak amplitude laser. The optimal rise time for achieving an adiabatic process with the same electric field intensity is different from one molecule to another. The molecular parameters determine how difficult it is to obtain an adiabatic process for specific laser pulses. A smaller rotational constant $B$ and a larger anisotropic polarizability $\Delta \alpha$ are beneficial to obtain a higher degree of orientation and results where the maximum degree of the field-free orientation is less sensitive to the fall time, however, also conditions where the adiabatic process is not easy to obtain. Further, with a sufficiently high laser intensity, the degree of orientation will increase with the value of $\Delta \alpha / B$ and is independent of the hyperpolarizability. The effect of the hyperpolarizability is to break the symmetry of the double-well potential for obtaining molecular orientation and a large absolute value of the hyperpolarizability is advantageous for achieving an adiabatic process.

In summary, for a specific molecule, we can choose a high laser intensity in order to obtain a high degree of molecular orientation, and increase the rise time for satisfying the adiabatic condition. On the other hand, the rise time and intensity of this truncated laser pulse are confined experimentally by its available energy [44] (see also Ref. [15]), and we can select a molecule with a small value of $\Delta \alpha / B$ and large absolute value of the hyperpolarizability for satisfying the adiabatic condition under specific laser parameters and obtain a relative high degree of field-free orientation if the fall time is small enough. The slowly rising pulse having relatively low peak intensity is beneficial for minimizing ionization.

Our analysis was based on the initial rotational state $J=0$ and can easily be extended to other rotational states. To that end, we note that state selection methods can be used to separate molecules with different initial states to avoid a significant decrease of the degree of molecular orientation [45].

\section{ACKNOWLEDGMENT}

This work was supported by the State Scholarships Fund of China Scholarship Council (No. 201906065022) and the Natural Science Foundation of Liaoning Province of China (No. 2019-ZD-0009).
[1] I. V. Litvinyuk, K. F. Lee, P. W. Dooley, D. M. Rayner, D. M. Villeneuve, and P. B. Corkum, Alignment-Dependent Strong Field Ionization of Molecules, Phys. Rev. Lett. 90, 233003 (2003).

[2] V. Aquilanti, M. Bartolomei, F. Pirani, D. Cappelletti, F. Vecchiocattivi, Y. Shimizu, and T. Kasai, Orienting and aligning molecules for stereochemistry and photodynamics, Phys. Chem. Chem. Phys. 7, 291 (2005).

[3] H. Stapelfeldt and T. Seideman, Colloquium: Aligning molecules with strong laser pulses, Rev. Mod. Phys. 75, 543 (2003).

[4] J. Itatani, J. Levesque, D. Zeidler, H. Niikura, H. Pépin, J.-C. Kieffer, P. B. Corkum, and D. M. Villeneuve, Tomographic imaging of molecular orbitals, Nature (London) 432, 867 (2004).
[5] X.-B. Bian and A. D. Bandrauk, Probing Nuclear Motion by Frequency Modulation of Molecular High-Order Harmonic Generation, Phys. Rev. Lett. 113, 193901 (2014).

[6] W. Boutu, S. Haessler, H. Merdji, P. Breger, G. Waters, M. Stankiewicz, L. Frasinski, R. Taieb, J. Caillat, A. Maquet et al., Coherent control of attosecond emission from aligned molecules, Nat. Phys. 4, 545 (2008).

[7] R. Velotta, N. Hay, M. B. Mason, M. Castillejo, and J. P. Marangos, High-Order Harmonic Generation in Aligned Molecules, Phys. Rev. Lett. 87, 183901 (2001).

[8] C. P. Koch, M. Lemeshko, and D. Sugny, Quantum control of molecular rotation, Rev. Mod. Phys. 91, 035005 (2019).

[9] B. Friedrich and D. Herschbach, Alignment and Trapping of Molecules in Intense Laser Fields, Phys. Rev. Lett. 74, 4623 (1995). 
[10] T. Seideman, Manipulating external degrees of freedom with intense light: Laser focusing and trapping of molecules, J. Chem. Phys. 106, 2881 (1997).

[11] H. Sakai, C. Safvan, J. J. Larsen, K. M. Hilligsøe, K. Hald, and H. Stapelfeldt, Controlling the alignment of neutral molecules by a strong laser field, J. Chem. Phys. 110, 10235 (1999).

[12] T. Seideman, Rotational excitation and molecular alignment in intense laser fields, J. Chem. Phys. 103, 7887 (1995).

[13] I. S. Averbukh and R. Arvieu, Angular Focusing, Squeezing, and Rainbow Formation in a Strongly Driven Quantum Rotor, Phys. Rev. Lett. 87, 163601 (2001).

[14] F. Rosca-Pruna and M. J. J. Vrakking, Experimental Observation of Revival Structures in Picosecond Laser-Induced Alignment of i2, Phys. Rev. Lett. 87, 153902 (2001).

[15] A. S. Chatterley, E. T. Karamatskos, C. Schouder, L. Christiansen, A. V. Jørgensen, T. Mullins, J. Küpper, and H. Stapelfeldt, Communication: Switched wave packets with spectrally truncated chirped pulses, J. Chem. Phys. 148, 221105 (2018).

[16] M. J. Vrakking and S. Stolte, Coherent control of molecular orientation, Chem. Phys. Lett. 271, 209 (1997).

[17] S. De, I. Znakovskaya, D. Ray, F. Anis, N. G. Johnson, I. A. Bocharova, M. Magrakvelidze, B. D. Esry, C. L. Cocke, I. V. Litvinyuk, and M. F. Kling, Field-Free Orientation of CO Molecules by Femtosecond Two-Color Laser Fields, Phys. Rev. Lett. 103, 153002 (2009).

[18] M. Machholm and N. E. Henriksen, Field-Free Orientation of Molecules, Phys. Rev. Lett. 87, 193001 (2001).

[19] S. Fleischer, Y. Zhou, R. W. Field, and K. A. Nelson, Molecular Orientation and Alignment by Intense Single-Cycle Thz Pulses, Phys. Rev. Lett. 107, 163603 (2011).

[20] K. Kitano, N. Ishii, and J. Itatani, High degree of molecular orientation by a combination of thz and femtosecond laser pulses, Phys. Rev. A 84, 053408 (2011).

[21] C.-C. Shu and N. E. Henriksen, Field-free molecular orientation induced by single-cycle thz pulses: The role of resonance and quantum interference, Phys. Rev. A 87, 013408 (2013).

[22] C. M. Dion, A. Keller, and O. Atabek, Optimally controlled field-free orientation of the kicked molecule, Phys. Rev. A 72, 023402 (2005).

[23] P. M. Kraus, D. Baykusheva, and H. J. Wörner, Two-Pulse Field-Free Orientation Reveals Anisotropy of Molecular Shape Resonance, Phys. Rev. Lett. 113, 023001 (2014).

[24] H. Yun, H. T. Kim, C. M. Kim, C. H. Nam, and J. Lee, Parity-selective enhancement of field-free molecular orientation in an intense two-color laser field, Phys. Rev. A 84, 065401 (2011).

[25] B. Friedrich and D. Herschbach, Manipulating molecules via combined static and laser fields, J. Phys. Chem. A 103, 10280 (1999).

[26] B. Friedrich and D. Herschbach, Enhanced orientation of polar molecules by combined electrostatic and nonresonant induced dipole forces, J. Chem. Phys. 111, 6157 (1999).

[27] J. J. Omiste and R. González-Férez, Nonadiabatic effects in long-pulse mixed-field orientation of a linear polar molecule, Phys. Rev. A 86, 043437 (2012).
[28] H. Tanji, S. Minemoto, and H. Sakai, Three-dimensional molecular orientation with combined electrostatic and elliptically polarized laser fields, Phys. Rev. A 72, 063401 (2005).

[29] J. L. Hansen, J. J. Omiste, J. H. Nielsen, D. Pentlehner, J. Küpper, R. González-Férez, and H. Stapelfeldt, Mixed-field orientation of molecules without rotational symmetry, J. Chem. Phys. 139, 234313 (2013).

[30] J. S. Kienitz, S. Trippel, T. Mullins, K. Długołecki, R. González-Férez, and J. Küpper, Adiabatic mixed-field orientation of ground-state-selected carbonyl sulfide molecules, ChemPhysChem 17, 3740 (2016).

[31] T. Seideman, Revival Structure of Aligned Rotational Wave Packets, Phys. Rev. Lett. 83, 4971 (1999).

[32] J. G. Underwood, M. Spanner, M. Y. Ivanov, J. Mottershead, B. J. Sussman, and A. Stolow, Switched Wave Packets: A Route to Nonperturbative Quantum Control, Phys. Rev. Lett. 90, 223001 (2003).

[33] T. Kanai and H. Sakai, Numerical simulations of molecular orientation using strong, nonresonant, two-color laser fields, J. Chem. Phys. 115, 5492 (2001).

[34] S. Guérin, L. P. Yatsenko, H. R. Jauslin, O. Faucher, and B. Lavorel, Orientation of Polar Molecules by Laser Induced Adiabatic Passage, Phys. Rev. Lett. 88, 233601 (2002).

[35] M. Muramatsu, M. Hita, S. Minemoto, and H. Sakai, Field-free molecular orientation by an intense nonresonant two-color laser field with a slow turn on and rapid turn off, Phys. Rev. A 79, 011403(R) (2009).

[36] K. Oda, M. Hita, S. Minemoto, and H. Sakai, All-Optical Molecular Orientation, Phys. Rev. Lett. 104, 213901 (2010).

[37] J. H. Mun and H. Sakai, Improving molecular orientation by optimizing relative delay and intensities of two-color laser pulses, Phys. Rev. A 98, 013404 (2018).

[38] S. Ramakrishna and T. Seideman, Intense Laser Alignment in Dissipative Media as a Route to Solvent Dynamics, Phys. Rev. Lett. 95, 113001 (2005).

[39] A. Messiah, Quantum Mechanics (North-Holland, 1962); translated by J. Potter (from French).

[40] D. Sugny, A. Keller, O. Atabek, D. Daems, C. M. Dion, S. Guérin, and H.-R. Jauslin, Reaching optimally oriented molecular states by laser kicks, Phys. Rev. A 69, 033402 (2004).

[41] S. Zhang, C. Lu, T. Jia, Z. Wang, and Z. Sun, Controlling fieldfree molecular orientation with combined single-and dual-color laser pulses, Phys. Rev. A 83, 043410 (2011).

[42] G. Maroulis and M. Menadakis, Polarizability and hyperpolarizability of COS and NNO, Chem. Phys. Lett. 494, 144 (2010).

[43] K. Sonoda, A. Iwasaki, K. Yamanouchi, and H. Hasegawa, Field-free molecular orientation of nonadiabatically aligned OCS, Chem. Phys. Lett. 693, 114 (2018).

[44] J. H. Mun, S. Minemoto, and H. Sakai, Development of a plasma shutter applicable to 100 -mj-class, 10-ns laser pulses and the characterization of its performance, Opt. Express 27, 19130 (2019).

[45] L. Holmegaard, J. H. Nielsen, I. Nevo, H. Stapelfeldt, F. Filsinger, J. Küpper, and G. Meijer, Laser-Induced Alignment and Orientation of Quantum-State-Selected Large Molecules, Phys. Rev. Lett. 102, 023001 (2009). 